\title{
Pyogenic liver abscess in non-liver cancer patients and liver cancer patients treated with TACE: etiological characteristics, treatment, and outcome analysis
}

\author{
Yiming Liu \\ Huazhong University of Science and Technology \\ Yanqiao Ren \\ Huazhong University of Science and Technology \\ Jun Chen \\ Fifth Hospital In Wuhan \\ Songlin Song \\ Huazhong University of Science and Technology \\ Chuansheng Zheng ( $\sim$ hqzcsxh@sina.com) \\ Huazhong University of Science and Technology
}

\section{Research Article}

Keywords: pyogenic liver abscess, liver cancer, transarterial chemoembolization, pathogenic bacteria

Posted Date: June 4th, 2021

DOl: https://doi.org/10.21203/rs.3.rs-558814/v1

License: (c) (i) This work is licensed under a Creative Commons Attribution 4.0 International License.

Read Full License 


\section{Abstract}

Background: The purpose of this study was to evaluate the clinical, laboratory and microbiological features, clinical outcomes, and prognosis of pyogenic liver abscess (PLA) in non-liver cancer (Non-LC) patients and liver cancer patients treated with transarterial chemoembolization (TACE, LC-TACE).

Methods: Clinical data of 48 consecutive PLA patients from January 2016 to December 2020 were retrospectively analyzed. The mortality was compared between the two groups of PLA patients, and risk factors for mortality were evaluated.

Results: A total of 48 PLA patients meeting the inclusion criteria were included in this study from January 2016 to December 2020, including 31 males and 17 females. Among them, there were 32 patients in the Non-LC group and 16 patients in the LC-TACE group. Fever and chills were the most common symptoms in both groups, followed by abdominal pain. Shock occurred in 2 patient in the Non-LC group and 3 patients in the LC-TACE group. The positive rate of pus culture in the Non-LC group was $87.5 \%$, among which the most common pathogen was Klebsiella pneumoniae $(50 \%, 14 / 28)$, and the positive rate of pus culture in the LC-TACE group was $81.3 \%$, among which the most common pathogen was Escherichia coli $(30.8 \%, 4 / 13)$. In the Non-LC group, 28 patients improved after treatment, 1 patient did not improve, and 3 patients died during hospitalization, with a mortality rate of 9.4\%. In the LC-TACE group, 9 cases improved after treatment, 3 cases did not improve, and 4 cases died during hospitalization, with a mortality rate of $25 \%$. Multivariate analysis revealed no independent prognostic factor associated with mortality. The cure time of the Non-LC group was 37.4 \pm 23.1 days (6-90 days), while that of the LC-TACE group was $91.5 \pm 49.7$ days (19-180 days), with a statistically significant difference between the two groups $(P<$ $0.001)$.

Conclusions: PLA of the Non-LC group and the LC-TACE group were different in terms of pathogenic bacteria and cure time, etc. For PLA after TACE, a more aggressive and comprehensive treatment should be considered.

\section{Introduction}

Pyogenic liver abscess (PLA) is an intrahepatic infection caused by suppurative bacteria that invade the liver, accounting for about $80 \%$ of all liver abscesses, and is commonly seen in patients with malnutrition and immunocompromised [1, 2]. An abscess occurs when normal liver clearance mechanisms are overwhelmed or when the system fails. Aplastic cholangitis and appendicitis are recognized causes of PLA, while the frequency of PLA caused by cholangitis and/or appendicitis has decreased dramatically with the use of effective imaging tools and the early administration of appropriate antibiotics [3]. However, a recent study [4] indicated that the incidence of the PLA has more than tripled in the past 35 years, suggesting other reasons for the increase.

Transarterial chemoembolization (TACE) has become a popular treatment of unresectable liver cancer and has been considered as an effective treatment modality [5-7]. It can target multiple lesions of liver 
cancer in a single treatment and can be used repeatedly in the same patient [8]. Of course, there are side effects after TACE, some of which can be life-threatening [9], including liver abscesses, a potentially fatal complication [10]. Similar to post-embolization syndrome, the most common complication after TACE, PLA also present with fever and right upper quadrant pain, but unlike post-embolization syndrome, PLA requires aggressive interventions, such as appropriate antibiotics and percutaneous catheter drainage [9]. Therefore, PLA caused by TACE in liver cancer patients should be paid more attention.

TACE can lead to the formation of PLA, which may be caused by many factors [11]: (1) bacterial infection superimposed upon embolization and necrosis; (2) intraoperative aseptic operation was not paid attention to; (3) patients with low immunity due to the immunosuppressive effect of chemotherapy drugs; (4) diabetic patients. Although many studies $[7,12,13]$ have reported the incidence, risk factors and treatment methods of PLA after TACE, as far as we know, no study has analyzed and compared the clinical characteristics and causative pathogen characteristics of PLA in non-liver cancer patients and liver cancer patients treated with TACE.

Hence, we conducted a retrospective study of PLA patients, including comparing the demographic characteristics, symptoms, clinical manifestations, laboratory and microbial pathogenic bacteria, clinical outcomes and mortality of PLA patients (non-liver cancer group and TACE treatment group) between two groups. Meanwhile, factors related to the mortality were also analyzed.

\section{Materials And Methods}

\section{Study Population}

The present retrospective study was conducted in accordance with the principles of the Declaration of Helsinki and received approval from the institutional review board of the Union Hospital, Tongji Medical College, Huazhong University of Science and Technology. Written informed consent was obtained from all patients.

We retrospectively analyzed the medical and microbiological records of all PLA patients from January 2016 to December 2020. A total of 48 patients who met the following criteria were included in our study $[14,15]$ : 1) patients who were confirmed to have PLA by percutaneous drainage or percutaneous aspiration; 2) imaging demonstrated that the abscess cavity was liquefied without rupture; 3 ) laboratory tests such as leukocytes, neutrophils and other indicators significantly increased; 4) no other extrahepatic abdominal abscesses were found. Exclusion criteria were: 1 ) incomplete clinical information; 2 ) amoebic liver abscess or infected liver cyst; 3) general infection; 4) Severe mental disorders are not compatible with treatment.

PLA patients meeting the inclusion criteria for this study were divided into two groups: patients with liver cancer receiving TACE (LC-TACE group) and patients without liver cancer (Non-LC group). Among them, there were 16 patients (mean age $18 \pm 1$ years, 18-56 years) in the LC-TACE group and 32 patients (mean age $19 \pm 2$ years, $17-42$ years) in the Non-LC group. 


\section{Treatments}

After diagnosis of PLA, all patients were given an intravenous drip of empirical broad-spectrum antibiotics until the availability of pus culture sensitivity report. In addition to intravenous antibiotics, invasive procedures such as percutaneous catheter drainage (PCD) are also the first treatment options to be considered. In general, an abscess $\geq 3 \mathrm{~cm}$ and characterized by a cystic appearance should be considered an indication of drainage [16]. PCD was performed under full aseptic precaution. After local anesthesia, the $21 \mathrm{G}$ Chiba needle was punctured into the abscess cavity under the guidance of ultrasound. The pus was sucked out with a syringe, the color and properties of the pus were observed, and the pus was sent to the laboratory for culture and drug sensitivity analysis. Draw out as much pus as possible. Then, a 10-F multipurpose drainage catheter was placed into the abscess cavity and a drainage bag was attached (Supplementary Figs. 1 and 2). Measure the drainage volume daily and flush the catheter with normal saline regularly to prevent the catheter from clogging.

\section{Microbiologic sample collection and cultivation}

The blood and pus of PLA patients were extracted for microbial cultures, and all samples were cultured under aerobic and anaerobic conditions. Blood samples from patients were collected under the following conditions: 1) body temperature was higher than $38^{\circ} \mathrm{C}$ or lower than $36^{\circ} \mathrm{C}$; 2) had chills; 3) leukocyte count was higher than $10 \times 10^{9} / \mathrm{L}$ or lower than $4 \times 10^{9} / \mathrm{L}$.

\section{Clinical Data and Definitions of Outcomes}

Demographic characteristics, etiological factors, co-existing conditions, number and location of abscesses, laboratory examination, diagnosis and treatment methods, treatment response, and mortality of PLA patients were analyzed. The 'cure' criteria were [1]: 1) the body temperature is normal for at least 3 days; 2) blood routine and inflammatory indexes were normal; 3 ) less than $5 \mathrm{ml}$ of drainage fluid per day. Meanwhile, we defined the drainage catheter removal time as the cure time. 'Uncured' was defined as no significant improvement or further deterioration of clinical symptoms after treatment, or 2 abnormal items in laboratory examination or imaging results despite improvement in symptoms.

\section{Statistical analysis}

SPSS software (version 24.0; IBM, Armonk, New York) was used for analyses. Quantitative data were represented by means \pm standard deviations and discrete variables were represented by proportion. We compared quantitative or discrete variables between the groups using the t-test, chi-square test, and ANOVA test. Factors associated with mortality were analyzed using binary logistic regression analysis. $P$ $<0.05$ (two-tailed) was considered statistically significant.

\section{Results}


From January 2016 to December 2020, a total of 48 PLA patients, including 31 males and 17 females, were included in this study after excluding patients who did not meet the inclusion criteria. Among them, there were 32 patients in the Non-LC group and 16 patients in the LC-TACE group (Table 1). In the Non-LC group, 17 patients had diabetes, while only 1 patient in the LC-TACE group had diabetes, showing a statistically significant difference between the two groups $(P=0.002)$. Although the maximum body temperature $\left(39.0 \pm 0.8^{\circ} \mathrm{C}\right.$ vs $\left.38.8 \pm 1^{\circ} \mathrm{C}\right)$ and maximum abscess diameter $(7.6 \pm 3.8 \mathrm{~cm}$ vs $7.3 \pm 2.6 \mathrm{~cm})$ of patients in the LC-TACE group were higher than those in the non-TACE group, there was no statistically significant difference between the two groups $(P>0.05)$. Most of the PLA in both groups were located in the right lobe of the liver, and most of the PLA were single abscess. Fever and chills were the most common symptom in both groups, followed by abdominal pain. Shock occurred in 2 patient in the Non-LC group and 3 patients in the LC-TACE group. 
Table 1

Baseline characteristics of PLA patients in the two groups

\begin{tabular}{|c|c|c|c|}
\hline Characteristics & $\begin{array}{l}\text { Non-LC group }(n=32) \\
(\text { No, \%; Mean } \pm \text { SD) }\end{array}$ & $\begin{array}{l}\text { LC-TACE group }(n=16) \\
(\text { No, \%; Mean } \pm \text { SD) }\end{array}$ & $\begin{array}{l}P \\
\text { value }\end{array}$ \\
\hline Age (years) & $58.4 \pm 12.6$ & $55.7 \pm 13.9$ & 0.548 \\
\hline Gender & & & 0.831 \\
\hline Male & $21(65.6 \%)$ & $10(62.5 \%)$ & \\
\hline Female & $11(34.4 \%)$ & $6(37.5 \%)$ & \\
\hline Abscess location & & & 0.605 \\
\hline Right lobe & $21(65.6 \%)$ & $9(56.3 \%)$ & \\
\hline Left lobe & $10(31.3 \%)$ & $6(37.5 \%)$ & \\
\hline Bilobar involvement & $2(6.3 \%)$ & $1(6.3 \%)$ & 1 \\
\hline Number of abscess & & & 0.433 \\
\hline 1 & $25(78.1 \%)$ & $14(87.5 \%)$ & \\
\hline$>1$ & $7(21.9 \%)$ & $2(12.5 \%)$ & \\
\hline $\begin{array}{l}\text { Maximal abscess } \\
\text { diameter }(\mathrm{cm})\end{array}$ & $7.3 \pm 2.6$ & $7.6 \pm 3.8$ & 0.762 \\
\hline \multicolumn{4}{|l|}{ Symptoms and signs } \\
\hline Fever/chills & $26(81.3 \%)$ & $14(87.5 \%)$ & 0.584 \\
\hline Abdominal pain & $13(40.6 \%)$ & $9(56.3 \%)$ & 0.306 \\
\hline Nausea/vomiting & $6(18.8 \%)$ & $2(12.5 \%)$ & 0.584 \\
\hline General weakness & $5(15.6 \%)$ & $2(12.5 \%)$ & 0.772 \\
\hline Diarrhoea & $3(9.4 \%)$ & $1(6.3 \%)$ & 0.712 \\
\hline Cough/dyspnoea & $2(6.3 \%)$ & 0 & 0.307 \\
\hline Pleuritic/chest pain & $2(6.3 \%)$ & 0 & 0.307 \\
\hline Shock & $2(6.3 \%)$ & $3(18.8 \%)$ & 0.181 \\
\hline Body temperature, ${ }^{\circ} \mathrm{C}$ & $38.8 \pm 1$ & $39.0 \pm 0.8$ & 0.518 \\
\hline \multicolumn{4}{|l|}{ Underlying disease } \\
\hline Diabetes mellitus & $17(53.1 \%)$ & $1(6.3 \%)$ & 0.002 \\
\hline
\end{tabular}

Note. PLA, Pyogenic liver abscess; Non-LC, Non liver cancer; LC-TACE, Liver cancer receiving TACE; SD, Standard deviation. 


\begin{tabular}{|lllr|}
\hline Characteristics & $\begin{array}{l}\text { Non-LC group }(\mathrm{n}=\mathbf{3 2}) \\
(\text { No, \%; Mean } \pm \text { SD) }\end{array}$ & $\begin{array}{l}\text { LC-TACE group }(\mathrm{n}=16) \\
(\text { No, \%; Mean } \pm \text { SD) }\end{array}$ & $\begin{array}{l}P \\
\text { value }\end{array}$ \\
\hline $\begin{array}{l}\text { Hepatobiliary and } \\
\text { pancreatic diseases }\end{array}$ & $12(37.5 \%)$ & $4(25 \%)$ & 0.386 \\
\hline Hepatobiliary calculi & $11(34.4 \%)$ & $3(18.8 \%)$ & 0.262 \\
\hline Hypertension & $6(18.8 \%)$ & $2(12.5 \%)$ & 0.584 \\
\hline $\begin{array}{l}\text { Note. PLA, Pyogenic liver abscess; Non-LC, Non liver cancer; LC-TACE, Liver cancer receiving TACE; SD, } \\
\text { Standard deviation. }\end{array}$ & & \\
\hline
\end{tabular}

\section{Microbiologic identifications and Laboratory examinations}

The positive rate of pus culture was $87.5 \%$ in the Non-LC group and $81.3 \%$ in the LC-TACE group, and there was no significant statistical difference between the two groups $(P=0.563)$ (Table 2). In the Non-LC group, among the 28 positive cases, $50 \%$ (14/28) of the pathogens were Klebsiella pneumoniae, followed by Viridians streptococcus, Escherichia coli and Pseudomonas aeruginosa; while in the LC-TACE group, among the 13 positive cases, the most common pathogens were Escherichia coli $(30.8 \%, 4 / 13)$, followed by Staphylococcus aureus, Viridians streptococcus, and Proteus mirabilis, and only 1 case was Klebsiella pneumoniae, the differences were statistically significant between the two groups $(P=0.009)$.

Table 2

Microbiological characteristics of two groups of PLA patients during hospitalization: pus culture

\begin{tabular}{|c|c|c|c|}
\hline Characteristics & $\begin{array}{l}\text { Non-LC group }(n=32) \\
(\text { No, \%) }\end{array}$ & $\begin{array}{l}\text { LC-TACE group }(n=16) \\
(\mathrm{No}, \%)\end{array}$ & $\begin{array}{l}P \\
\text { value }\end{array}$ \\
\hline Positive growth & $28(87.5 \%)$ & $13(81.3 \%)$ & 0.563 \\
\hline Polymicrobial growth & $2(7.1 \%)$ & $1(7.7 \%)$ & 0.95 \\
\hline Klebsiella pneumonia & $14(50 \%)$ & $1(7.7 \%)$ & 0.009 \\
\hline $\begin{array}{l}\text { Viridians } \\
\text { streptococcus }\end{array}$ & $5(17.9 \%)$ & $2(15.4 \%)$ & 0.845 \\
\hline Escherichia coli & $3(10.7 \%)$ & $4(30.8 \%)$ & 0.112 \\
\hline $\begin{array}{l}\text { Pseudomonas } \\
\text { aeruginosa }\end{array}$ & $3(10.7 \%)$ & $1(7.7 \%)$ & 0.762 \\
\hline $\begin{array}{l}\text { Staphylococcus } \\
\text { aureus }\end{array}$ & $2(7.1 \%)$ & $3(23.1 \%)$ & 0.297 \\
\hline Proteus mirabilis & $1(3.6 \%)$ & $2(15.4 \%)$ & 0.176 \\
\hline
\end{tabular}


Compared with the LC-TACE group, patients in the Non-LC group had higher inflammatory biomarkers, including leukocytes ( $13.5 \pm 6.8$ vs $11.5 \pm 6.8, P=0.4)$, neutrophils ( $11.7 \pm 6.7$ vs $10.2 \pm 6.8, P=0.509)$, Creactive protein $(142.7 \pm 76.6$ vs $121.6 \pm 56.7, P=0.388)$, and procalcitonin $(20.1 \pm 28.8$ vs $6.4 \pm 7.4, P=$ $0.102)$, but there was no statistically significant difference between the two groups. After treatment of PLA, C-reactive protein (Non-LC group $89.4 \pm 64.1$ vs $80.3 \pm 41.5, P=0.644$ ) and procalcitonin (Non-LC group $7.9 \pm 21.1$ vs $2.3 \pm 3.5, P=0.352$ ) were significantly decreased in both groups, and there was no significant difference between the two groups. Meanwhile, alanine transaminase (ALT, $65.4 \pm 47.6$ vs 48.7 $\pm 35.7, P=0.275$ ) and aspartate aminotransferase (AST, $55.7 \pm 50.0$ vs $47.9 \pm 23.4, P=0.601$ ) in the NonLC group were higher than those in the LC-TACE group, while albumin $(28.2 \pm 5.1$ vs $29.6 \pm 3.5, P=0.334)$ and total bilirubin $(22.3 \pm 17.0$ vs $24.4 \pm 14.8, P=0.717)$ were lower than those in the LC-TACE group, and there was no significant difference in liver function between the two groups. Although PLA patients had elevated inflammatory biomarkers and abnormal liver function, the mean renal function including blood urea nitrogen and creatinine were normal in both groups (Table 3 ).

Table 3

Laboratory results of two groups of PLA patients

\begin{tabular}{|c|c|c|c|}
\hline Characteristics & $\begin{array}{l}\text { Non-LC group }(n=32) \\
(\text { Mean } \pm \text { SD) }\end{array}$ & $\begin{array}{l}\text { LC-TACE group }(n=16) \\
(\text { Mean } \pm \text { SD) }\end{array}$ & $\begin{array}{l}P \\
\text { value }\end{array}$ \\
\hline WBCc (X109/L) & $13.47 \pm 6.76$ & $11.50 \pm 6.81$ & 0.4 \\
\hline $\begin{array}{l}\text { Neutrophil count } \\
(\mathrm{X} 109 / \mathrm{L})\end{array}$ & $11.74 \pm 6.68$ & $10.21 \pm 6.75$ & 0.509 \\
\hline AST ( IU/L) & $55.7 \pm 50.0$ & $47.9 \pm 23.4$ & 0.601 \\
\hline $\operatorname{ALT}(\mathrm{IU} / \mathrm{L})$ & $65.36 \pm 47.64$ & $48.69 \pm 35.67$ & 0.275 \\
\hline $\begin{array}{l}\text { Prothrombin time, } \\
\text { INR }\end{array}$ & $14.4 \pm 2.7$ & $14.6 \pm 1.2$ & 0.81 \\
\hline $\begin{array}{l}\text { Total bilirubin } \\
(\mu \mathrm{mol} / \mathrm{L})\end{array}$ & $22.34 \pm 16.96$ & $24.37 \pm 14.82$ & 0.717 \\
\hline BUN (mmol/L) & $5.85 \pm 2.79$ & $6.97 \pm 5.24$ & 0.394 \\
\hline $\mathrm{Cr}(\mu \mathrm{mol} / \mathrm{L})$ & $59.78 \pm 13.24$ & $74.68 \pm 47.38$ & 0.286 \\
\hline CRP (mg/L) & $142.7 \pm 76.6$ & $121.6 \pm 56.7$ & 0.388 \\
\hline ALP (U/L) & $251.56 \pm 161.48$ & $212.77 \pm 90.80$ & 0.429 \\
\hline PCT $(\mu \mathrm{g} / \mathrm{L})$ & $20.1 \pm 28.8$ & $6.4 \pm 7.4$ & 0.102 \\
\hline Albumin (g/dL) & $28.204 \pm 5.08$ & $29.58 \pm 3.52$ & 0.334 \\
\hline \multicolumn{4}{|c|}{$\begin{array}{l}\text { Note. PLA, Pyogenic liver abscess; Non-LC, Non liver cancer; LC-TACE, Liver cancer receiving TACE; SD, } \\
\text { Standard deviation; WBCc, White blood cell count; ALT, Alanine aminotransferase; AST, Aspartate } \\
\text { aminotransferase; BUN, Blood urea nitrogen; Cr, Creatinine; CRP, Cross-reacting protein; ALP, Alkaline } \\
\text { phosphatase; PCT, Procalcitonin. }\end{array}$} \\
\hline
\end{tabular}




\section{Treatment outcomes and risk factors for mortality}

All patients were treated with intravenous sensitive antibiotics while receiving PCD. Of the patients in the Non-LC group, 62.5\% (20/32) received combined antibiotic treatment, i.e., third-generation cephalosporins or carbapenems combined with fluoroquinolones and metronidazole, and only 12 patients received single antibiotic treatment. Of the patients in the LC-TACE group, $62.5 \%(10 / 16)$ were treated with combination antibiotics and 6 patients were treated with single antibiotic.

In the Non-LC group, 28 patients improved after treatment, 1 patient did not improve, and 3 patients died during hospitalization, with a mortality rate of $9.4 \%$. In the LC-TACE group, 9 cases improved after treatment, 3 cases did not improve, and 4 cases died during hospitalization, with a mortality rate of $25 \%$. Univariate analysis (Table 4) demonstrated that leukocyte count (HR, 1.223; 95\% Cl: 1.040, 1.439, $P=$ $0.015)$, neutrophils count (HR, $1.220 ; 95 \% \mathrm{Cl}: 1.041,1.431, P=0.014)$, and total bilirubin (HR, $1.042 ; 95 \% \mathrm{Cl}$ : $0.993,1.093, P=0.095)$ were associated with mortality. However, when these three factors were included in the multivariate analysis (Table 5), there was no independent prognostic factor associated with mortality. The cure time of the Non-LC group was $37.4 \pm 23.1$ days (6-90 days), while that of the LC-TACE group was $91.5 \pm 49.7$ days (19-180 days), with a statistically significant difference between the two groups $(P<0.001)$. 
Table 4

Univariate analysis of prognostic factors for mortality of PLA patients in two groups

\begin{tabular}{|c|c|c|}
\hline Variables & HR (95\% Cl) & $P$ value \\
\hline Age (y) & $1.017(0.942,1.098)$ & 0.663 \\
\hline \multicolumn{3}{|l|}{ Gender } \\
\hline Female & 1 & \\
\hline Male & $0.333(0.048,2.297)$ & 0.265 \\
\hline \multicolumn{3}{|l|}{ Abscess location } \\
\hline Bilobar involvement & 1 & \\
\hline Right lobe & $0.087(0.004,1.981)$ & 0.126 \\
\hline Left lobe & $0.222(0.009,5.275)$ & 0.352 \\
\hline \multicolumn{3}{|l|}{ Number of abscess } \\
\hline$>1$ & 1 & \\
\hline 1 & - & 0.999 \\
\hline Maximal abscess diameter $(\mathrm{cm})$ & $1.093(0.798,1.498)$ & 0.580 \\
\hline Body temperature, ${ }^{\circ} \mathrm{C}$ & $0.825(0.297,2.291)$ & 0.712 \\
\hline \multicolumn{3}{|l|}{ Pus culture } \\
\hline No bacteria & 1 & \\
\hline Positive growth & $0.552(0.049,6.254)$ & 0.631 \\
\hline WBCc (X109/L) & $1.223(1.040,1.439)$ & 0.015 \\
\hline Neutrophil count (X109/L) & $1.220(1.041,1.431)$ & 0.014 \\
\hline ALT(IU/L) & $0.996(0.972,1.019)$ & 0.715 \\
\hline AST ( IU/L) & $0.992(0.961,1.023)$ & 0.590 \\
\hline Prothrombin time, INR & $1.506(0.830,2.734)$ & 0.178 \\
\hline Total bilirubin $(\mu \mathrm{mol} / \mathrm{L})$ & $1.042(0.993,1.093)$ & 0.095 \\
\hline BUN (mmol/L) & $1.133(0.917,1.399)$ & 0.247 \\
\hline $\mathrm{Cr}(\mu \mathrm{mol} / \mathrm{L})$ & $1.019(0.993,1.046)$ & 0.154 \\
\hline
\end{tabular}

Note. PLA, Pyogenic liver abscess; HR, Hazard ratio; Cl confidence interval; WBCc, White blood cell count; ALT, Alanine aminotransferase; AST, Aspartate aminotransferase; BUN, Blood urea nitrogen; $\mathrm{Cr}$, Creatinine; CRP, Cross-reacting protein; ALP, Alkaline phosphatase; PCT, Procalcitonin; LC-TACE, Liver cancer receiving TACE; Non-LC, Non liver cancer. 


\begin{tabular}{|llc|}
\hline Variables & HR $(95 \%$ Cl) & Pvalue \\
\hline CRP $(\mathrm{mg} / \mathrm{L})$ & $0.985(0.966,1.003)$ & 0.109 \\
\hline ALP $(\mathrm{U} / \mathrm{L})$ & $0.998(0.991,1.006)$ & 0.665 \\
\hline PCT $(\mu \mathrm{g} / \mathrm{L})$ & $0.978(0.915,1.046)$ & 0.519 \\
\hline Albumin $(\mathrm{g} / \mathrm{dL})$ & $0.867(0.692,1.086)$ & 0.213 \\
\hline Group & 1 & 0.210 \\
\hline LC-TACE & $0.290(0.042,2.011)$ & \\
\hline Non-LC & & \\
\hline $\begin{array}{l}\text { Note. PLA, Pyogenic liver abscess; HR, Hazard ratio; Cl confidence interval; WBCc, White blood cell } \\
\text { count; ALT, Alanine aminotransferase; AST, Aspartate aminotransferase; BUN, Blood urea nitrogen; Cr, } \\
\begin{array}{l}\text { Creatinine; CRP, Cross-reacting protein; ALP, Alkaline phosphatase; PCT, Procalcitonin; LC-TACE, Liver } \\
\text { cancer receiving TACE; Non-LC, Non liver cancer. }\end{array}\end{array}$ \\
\hline
\end{tabular}

Table 5

Multivariate analysis of prognostic factors for mortality of PLA patients in two groups

\begin{tabular}{|lll|}
\hline Variables & HR $(95 \%$ Cl $)$ & $P$ value \\
\hline WBCc $(X 109 / L)$ & $2.098(0.298,14.757)$ & 0.457 \\
\hline Neutrophil count $(\mathrm{X} 109 / \mathrm{L})$ & $0.585(0.086,3.982)$ & 0.584 \\
\hline Total bilirubin $(\mu \mathrm{mol} / \mathrm{L})$ & $1.042(0.984,1.104)$ & 0.161 \\
\hline $\begin{array}{l}\text { Note. PLA, Pyogenic liver abscess; HR, Hazard ratio; Cl confidence interval; WBCc, White blood cell } \\
\text { count. }\end{array}$ & \\
\hline
\end{tabular}

\section{Discussion}

To our knowledge, this is the first large population-based retrospective study to investigate the clinical and microbiological characteristics of PLA patients in the non-TACE group and the LC-TACE group, as well as to evaluate risk factors that may affect the mortality of PLA patients. Currently, many studies [9, $12,13]$ have only reported the clinical and microbiological characteristics of PLA patients after TACE. However, this study comprehensively compared the differences between the non-LC group and the LCTACE group, providing a new perspective on the mortality, clinical and epidemiological characteristics of the PLA.

In this study, the average age of patients in both groups was greater than 55 years old. Similar to other studies $[15,17]$, most of PLA patients in the two groups in this study were middle-aged and elderly, which 
suggested that PLA could be considered as a geriatric disease. A series of reports [17-19] have all confirmed the relationship between diabetes and PLA, which may be able to explain that $53.1 \%$ of the patients in the Non-LC group in this study had diabetes, while only one patient (6.3\%) in the TACE group had diabetes, indicating that there were significant differences between the two groups in terms of comorbidities. In this study, the most common symptom of patients in the two groups was fever and chills, followed by abdominal pain, which was basically consistent with other studies. In this study, the most common symptom of PLA patients in the two groups was fever and chills, followed by abdominal pain, which was basically consistent with previous studies $[20,21]$.

TACE can improve survival in liver cancer patients by combining targeted chemotherapy with ischemic necrosis caused by arterial embolization [22]. However, PLA is a serious postoperative complication, which deserves our attention. It is reported that the mortality rate of PLA patients after TACE ranged from $13.3-50 \%$, in this study, the mortality rate of patients in the LC-TACE group was $25 \%$, which was consistent with previous report [23]. The mortality rate of patients in the LC-TACE group was significantly higher than that in the Non-LC group. Unlike other studies [1], multivariate analysis in this study revealed that no risk factors were associated with mortality. Meanwhile, the cure time of patients in the LC-TACE group was also significantly longer than that in the Non-LC group, which may be due to the following reasons [7, 24, 25]: 1) Both tumor and TACE can lead to immunodeficiency in patients in the LC-TACE group, which can lead to patient death and prolonged treatment time, meanwhile, the hepatic ischemia and hypoxia induced by TACE provide a good environment for the growth of bacteria; 2) Some patients with fever after TACE were mistaken for postembolism syndrome, and dexamethasone was routinely applied, which accelerated the progress of the PLA; 3 ) Patients are unable to receive antitumor therapy during the treatment of PLA, leading to the progression of liver cancer, which hastens the patient's death. At the same time, the embolization leads to capillary blockage, and antibiotics cannot reach the abscess cavity. This may also explain the higher mean body temperature and more shock cases in the LC-TACE group. Therefore, we should attach great importance to the PLA after TACE and actively conduct abscess drainage.

In this study, the inflammatory biomarkers (leukocyte, neutrophils, C-reactive protein and procalcitonin) in the Non-LC group were higher than those in the LC-TACE group, suggesting that tumor itself may hinder the rise of inflammatory biomarkers. In addition, C-reactive protein and procalcitonin were significantly reduced in both groups after treatment without statistical difference, indicating that there was no significant difference in overall outcomes between the two groups using similar treatment regimens. Therefore, the bacterial etiology of liver abscesses may be different between the two groups, but the treatment of PLA is not substantially different in nature. Furthermore, AST, ALT and albumin in the Non-LC group were worse than those in the LC-TACE group, which indicated that PLA had a great influence on the liver function of patients.

It is reported that the prevalence of PLA caused by Klebsiella pneumoniae is on the rise worldwide [26, 27]. In this study, the PLA of $50 \%$ of patients in the non-LC group was caused by Klebsiella pneumoniae, while only 1 case in the LC-TACE group was caused by Klebsiella pneumoniae. Different from the Non-LC 
group, the most common pathogen of pus culture in the LC-TACE group was Escherichia coli (30.8\%), which was consistent with the often encountered bacterium of PLA after TACE reported by other studies $[28,29]$. This indicates that the pathogenic bacteria of PLA after TACE are different from those of PLA in non-liver cancer patients.

This study has its limitations. This was a single-center retrospective study with inherent flaws and a limited sample size. Therefore, it is necessary to conduct a multi-center large-sample study to evaluate the clinical characteristics, microbiological characteristics and treatment outcomes of the two groups of patients.

\section{Conclusions}

In this study, baseline characteristics, microbiological characteristics, and laboratory test results of patients in the Non-LC group and the LC-TACE group were compared in detail, and the prognosis of patients in the two groups was analyzed, and the differences between the two groups were comprehensively evaluated. For PLA patients after TACE, due to factors such as liver cancer and TACE, the treatment of PLA should be more aggressive and comprehensive.

\section{Declarations}

\section{Ethics approval and consent to participate}

The present retrospective study was conducted in accordance with the principles of the Declaration of Helsinki and received approval from the institutional review board of the Union Hospital, Tongji Medical College, Huazhong University of Science and Technology. Written informed consent was obtained from all patients.

\section{Consent for publication}

Not applicable

\section{Availability of data and materials}

The data analysed during this study are avaliable from the electrical medical database of Union Hospital, Tongji Medical college, Huazhong University of Science and Technology. Please contact the author Chuansheng Zheng (hqzcsxh@sina.com) upon reasonable requests.

\section{Competing interests}

The authors declare that they have no competing interests

\section{Funding}


This work was supported by grant from National Nature Science Foundation of China (81873919 and 81801810), Free Innovation Pre-research Fund of Union Hospital, Tongji Medical College, Huazhong University of Science and Technology (02.03.2019-157).

\section{Authors' contributions}

YML, YQR, and JC collected the patients' data, YML drafted the manuscript. YQR and JC revised the manuscript. SLS and CSZ analyzed and interpreted the data. SLS made substantial contributions to the conception of the work. SLS and CSZ made substantial contributions to the design of the work, and have revised the manuscript substantively. All authors read and approved the final manuscript.

\section{Acknowledgements}

We thank to all the medical workers in our department for their assistance with the study, without their encouragement and assistance this thesis would not have been completed.

\section{Authors' information}

${ }^{1}$ Department of Radiology, Union Hospital, Tongji Medical College, Huazhong University of Science and Technology, Wuhan 430022, China.

${ }^{2}$ Hubei Provinve Key Laboratory of Molecular Imaging, Wuhan 430022, China.

${ }^{3}$ Department of Radiology, FIFTH HOSPITAL IN WUHAN, 430050, China.

${ }^{4}$ Department of Radiology, Union Hospital, Tongji Medical College and Wuhan National Laboratory for Optoelectronics, Huazhong University of Science and Technology.

\section{References}

1. YinD, JiC, Zhang S, et al. Clinical characteristics and management of 1572 patients with pyogenic liver abscess: A 12-year retrospective study. Liver Int. 2021; 41: 810-818.

2. MohanBP, Meyyur AravamudanV, Khan SR, et al. Prevalence of colorectal cancer in cryptogenic pyogenic liver abscess patients. Do they need screening colonoscopy? A systematic review and meta-analysis. Dig Liver Dis. 2019; 51: 1641-1645.

3. Cohen JL, Martin FM, Rossi R, Schoetz D. Liver abscess. The need for complete gastrointestinal evaluation. Arch Surg. 1989; 124: 561-564.

4. Sharma A, Mukewar S, Mara KC, et al. Epidemiologic factors, clinical presentation, causes, and outcomes of liver abscess: a 35-year Olmsted county study. Mayo Clin Proc Innov Qual Outcomes. 2018; 2: 16-25. 
5. Cammà C, Schepis F, Orlando A, et al. Transarterial chemoembolization for unresectable hepatocellular carcinoma: meta-analysis of randomized controlled trials. Radiology. 2002; 224:4754.

6. Llovet JM, Bruix J. Systematic review of randomized trials for unresectable hepatocellular carcinoma: chemoembolization improves survival. Hepatology. 2003; 37:429-442.

7. SunW, XuF, Li X, Li CR. A Case Series of Liver Abscess Formation after Transcatheter Arterial Chemoembolization for Hepatic Tumors. Chin Med J (Engl). 2017; 130: 1314-1319.

8. Xia J, Ren Z, Ye S, et al. Study of severe and rare complications of transarterial chemoembolization (TACE) for liver cancer. Eur J Radiol. 2006; 59: 407-12.

9. ShinJU, KimKM, Shin SW, et al. A prediction model for liver abscess developing after transarterial chemoembolization in patients with hepatocellular carcinoma. Dig Liver Dis. 2014; 46: 813-817.

10. Mezhir JJ, Fong Y, Fleischer D, et al. Pyogenic abscess after hepatic artery embolization: a rare but potentially lethal complication. Journal of Vascular and Interventional Radiology. 2011; 22: 177-82.

11. Lv WF, Lu D, He YS, et al. Liver abscess formation following transarterial chemoembolization: Clinical features, risk factors, bacteria spectrum, and percutaneous catheter drainage. Medicine (Baltimore). 2016; 95: e3503.

12. WooS, ChungJW, Hur S, et al. Liver abscess after transarterial chemoembolization in patients with bilioenteric anastomosis: frequency and risk factors. AJR Am J Roentgenol. 2013; 200: 1370-1377.

13. JiaZ, TuJ, Cao C, et al. Liver abscess following transarterial chemoembolization for the treatment of hepatocellular carcinoma: A retrospective analysis of 23 cases. J Cancer Res Ther. 2018; 14: S628S633.

14. HuMJ, YuYX, Fan YF, Hu CH. CT-based radiomics model to distinguish necrotic hepatocellular carcinoma from pyogenic liver abscess. Clin Radiol. 2021; 76:161.e11-161.e17.

15. ZhouY, LuG, Bai Y, et al. Retrospective study of characteristics and management of pyogenic liver abscess during 5 years' experience. Int J Clin Exp Pathol. 2021; 14: 252-260.

16. LeeCH,Jo HG, Cho EY, et al. Maximal diameter of liver abscess independently predicts prolonged hospitalization and poor prognosis in patients with pyogenic liver abscess. BMC Infect Dis. 2021; 21: 171.

17. TianLT, YaoK, Zhang XY, et al. Liver abscesses in adult patients with and without diabetes mellitus: an analysis of the clinical characteristics, features of the causative $p$ athogens, outcomes and predictors of fatality: a report based on a large population, retrospective study in China. Clin Microbiol Infect. 2012; 18: E314-30.

18. Han SH. Review of hepatic abscess from Klebsiella pneumoniae. An association with diabetes mellitus and septic endophthalmitis. West J Med. 1995; 162: 220-224.

19. Foo NP, Chen KT, Lin HJ, Guo HR. Characteristics of pyogenic liver abscess patients with and without diabetes mellitus. Am J Gastroenterol. 2010; 105: 328-335. 
20. Kong H, Yu F, Zhang W et al. Clinical and microbiological characteristics of pyogenic liver abscess in a tertiary hospital in East China. Medicine (Baltimore). 2017; 96: e8050.

21. Ali AH, Smalligan RD, Ahmed M et al. Pyogenic liver abscess and the emergence of Klebsiella as an etiology: a retrospective study. Int J Gen Med. 2013; 7: 37-42.

22. RenY, CaoY, Ma H, et al. Improved clinical outcome using transarterial chemoembolization combined with radiofrequency ablation for patients in Barcelona clinic liver cancer stage $\mathrm{A}$ or $\mathrm{B}$ hepatocellular carcinoma regardless of tumor size: results of a single-center retrospective case control study. BMC Cancer. 2019; 19: 983.

23. $\mathrm{Li} \mathrm{JH}$, Yao RR, Shen HJ, et al. Clostridium perfringens infection after transarterial chemoembolization for large hepatocellular carcinoma. World J Gastroenterol. 2015; 21: 4397-401.

24. Pua U, Merkle EM. Case report. Spontaneous cholecystocolic fistula and locoregional liver tumour ablation: A cautionary tale. Br J Radiol. 2011; 84: e243-5.

25. ArslanM,Degirmencioglu S. Liver abscesses after transcatheter arterial embolization. J Int Med Res. 2019; 47: 1124-1130.

26. Rahimian J, Wilson T, Oram V, Holzman RS. Pyogenic liver abscess: recent trends in etiology and mortality. Clin Infect Dis. 2004; 39: 1654-1659.

27. Lederman ER, Crum NF. Pyogenic liver abscess with a focus on Klebsiella pneumoniae as a primary pathogen: an emerging disease with unique clinical characteristics. Am J Gastroenterol. 2005; 100 : 322-331.

28. OngGY,Changchien CS, Lee CM, et al. Liver abscess complicating transcatheter arterial embolization: a rare but serious complication. A retrospective study after 3878 procedures. Eur J Gastroenterol Hepatol. 2004; 16: 737-742.

29. LvWF,Lu D, He YS, et al. Liver Abscess Formation Following Transarterial Chemoembolization: Clinical Features, Risk Factors, Bacteria Spectrum, and Percutaneous Catheter Drainage. Medicine (Baltimore). 2016; 95: e3503.

\section{Figures}


A

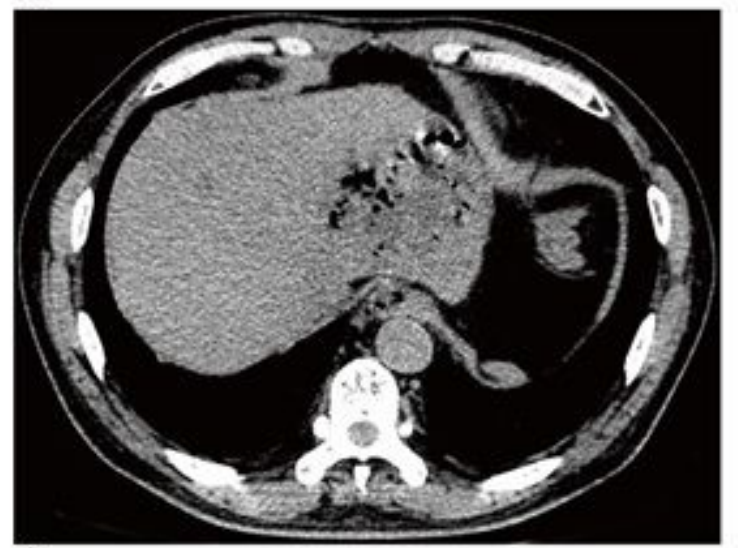

B

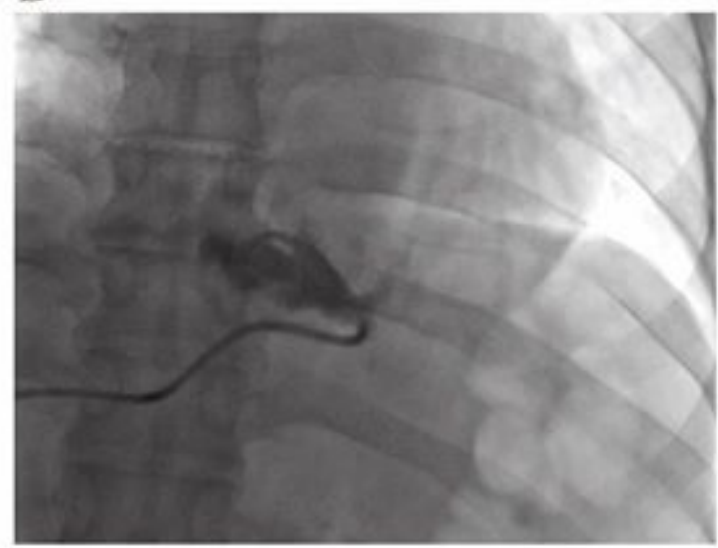

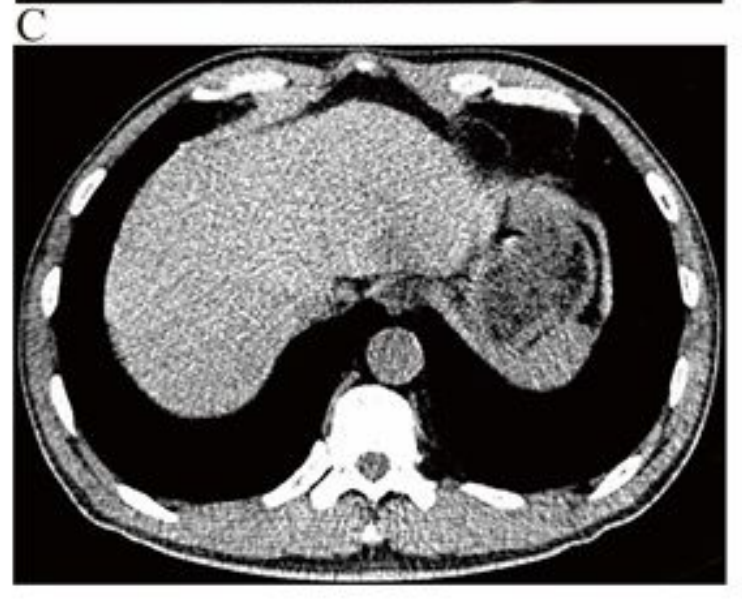

Figure 1

A 65-year-old male with type 2 diabetes was admitted with high fever, abdominal pain, and malignant vomiting with a maximum temperature of $39.6^{\circ} \mathrm{C}$. The CT scan showed a $7.6 \mathrm{~cm}$ diameter hypodense shadow with gas in the left lateral lobe of the liver (A). The abscess was treated with antibiotics and drained (B). Three months after treatment, CT showed the absence of low-density shadows (C). 

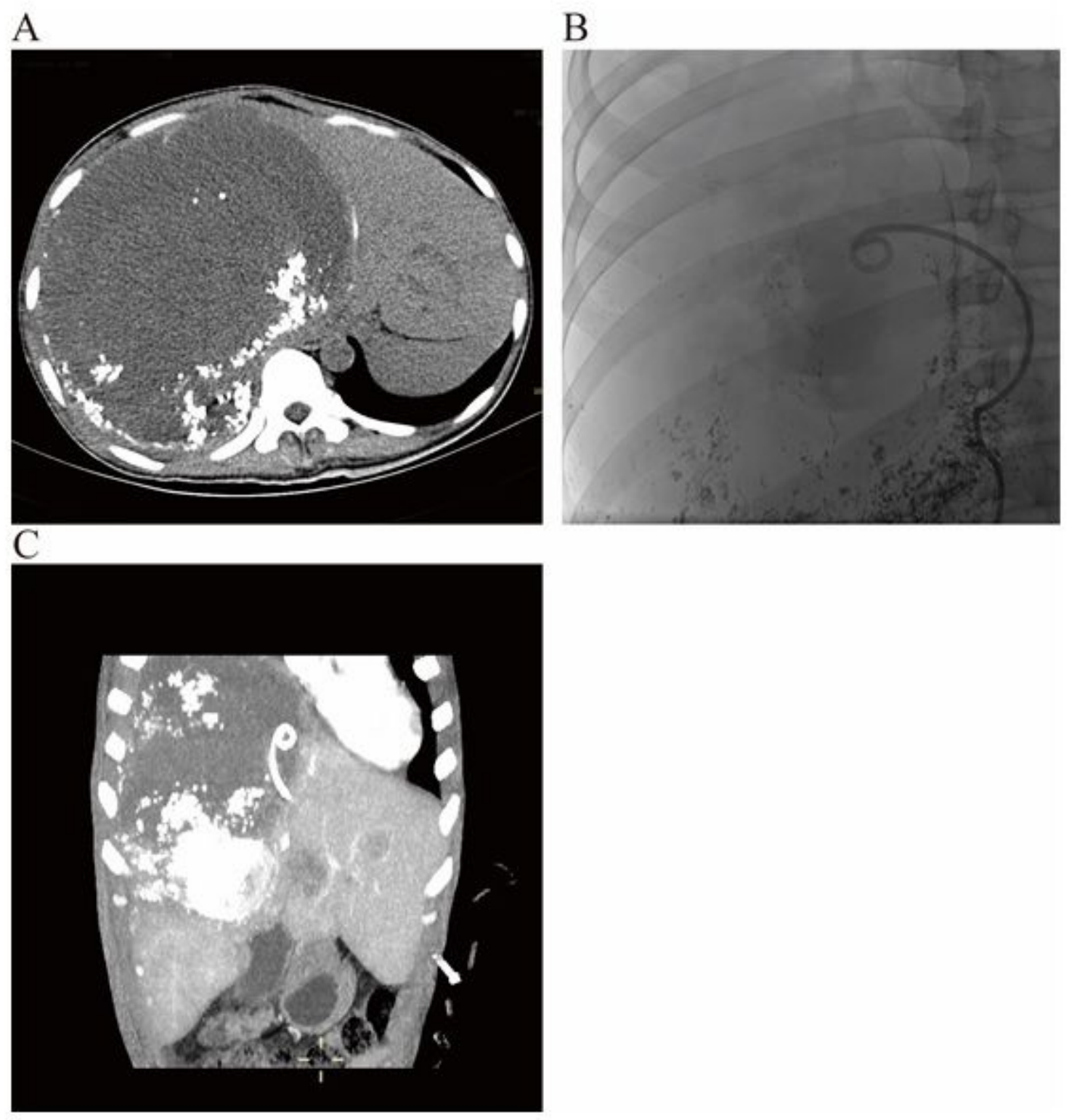

\section{Figure 2}

A 30-year-old male HCC patient developed high fever, chill, and abdominal pain 3 days after TACE, with a maximum body temperature of $39.4^{\circ} \mathrm{C}$. The CT scan indicated a large low-density shadow in the right lobe of the liver (A). The abscess was actively drained while antibiotic treatment was given (B). The CT scan 1 week after drainage demonstrated that the drainage tube was in good position (C). 